\title{
Synthesis of a novel 89Zr labeled HER2 affibody and its application study in tumor PET imaging
}

\author{
Yuping Xu \\ Jiangsu Institute of Nuclear Medicine \\ Lizhen Wang \\ Jiangsu Institute of Nuclear Medicine \\ Donghui Pan \\ Jiangsu Institute of Nuclear Medicine \\ Junjie Yan \\ Jiangsu Institute of Nuclear Medicine \\ Xinyu Wang \\ Jiangsu Institute of Nuclear Medicine \\ Runlin Yang \\ Jiangsu Institute of Nuclear Medicine \\ Mingzhu Li \\ Inner Mongolia Medical University \\ Yu Liu \\ Nanjing Medical University \\ Min Yang ( $\sim$ yangmin@jsinm.org )
}

\section{Original research}

Keywords: Human epidermal growth factor receptor-2, PET, affibody, Zirconium-89

Posted Date: June 29th, 2020

DOI: https://doi.org/10.21203/rs.3.rs-18136/v4

License: (c) (i) This work is licensed under a Creative Commons Attribution 4.0 International License. Read Full License

Version of Record: A version of this preprint was published at EJNMMI Research on June 3rd, 2020. See the published version at https://doi.org/10.1186/s13550-020-00649-7. 


\section{Abstract}

Background: Human epidermal growth factor receptor-2 (HER2) is an essential biomarker for tumor treatment. Affibody is an ideal vector for preparing HER2 specific probes because of high affinity and rapid clearance from normal tissues etc. Zirconium-89 is a PET imaging isotope with a long half-life and suitable for monitoring biological processes for more extended periods. In this study, a novel ${ }^{89} \mathrm{Zr}$-labeled HER2 affibody, $\left[{ }^{89} \mathrm{Zr}\right.$ ZZr-DFO-MAL-Cys-MZHER2, was synthesized, and its imaging characters were also assessed.

Results: The precursor, DFO-MAL-Cys-MZHER2, was obtained with a yield of nearly $50 \%$. The radiochemical yield of $\left[{ }^{89} \mathrm{Zr}\right] \mathrm{Zr}$-DFO-MAL-Cys-MZHER2 was $90.2 \pm 1.9 \%$, and the radiochemical purity was higher than $95 \%$. The total synthesis time was only 30 minutes. The probe was stable in PBS and serum. The tracer accumulated in HER2 overexpressing human ovarian cancer SKOV-3 cells. In vivo studies in mice bearing tumors showed that the probe highly retained in SKOV-3 xenografts even for 48 hours. The tumors were visualized with good contrast to normal tissues. ROI analysis revealed that the average uptake values in the tumor were greater than $5 \% \mathrm{IA} / \mathrm{g}$ during 48 hours postinjection. On the contrary, the counterparts of MCF-7 tumors kept low levels $\varangle \sim 1 \% \mathrm{IA} / \mathrm{g} \rrbracket$. The outcome was consistent with the immunohistochemical analysis and ex vivo autoradiography. The probe quickly cleared from the normal organs except kidneys and mainly excreted through the urinary system.

Conclusion: The novel HER2 affibody for PET imaging was easily prepared with satisfactory labeling yield and radiochemical purity. $\left[{ }^{89} \mathrm{Zr}\right.$ ]Zr-DFO-MAL-Cys-MZHER2 is a potential candidate for detecting HER2 expression. It may play specific roles in clinical cancer theranostics.

\section{Introduction}

Targeting of a biomarker in cancers with specific agents is a promising strategy in the management of malignancies. ${ }^{[1]}$ Human epidermal growth factor receptor type 2 (HER2) is a 185-kDa transmembrane protein, which belongs to the family of receptor tyrosine kinases. It involves in the signal transduction pathways regulating cell motility and proliferation. HER2 is an essential clinical tumor biomarker since it is overexpressed in various solid tumors, including ovarian, gastric, breast cancers, etc and associated with aggressiveness, recurrence, and reduced survival. ${ }^{[2-4]}$

Monoclonal antibodies, including trastuzumab and pertuzumab etc have been used for therapy of HER2-positive cancers. ${ }^{[5-7]}$ Current selection of patients for targeted therapy is mainly dependent on the status of HER2, which was determined by biopsy using immunohistochemistry or fluorescence in situ hybridization. ${ }^{[8]}$ However, the invasive method may not be reliable because the HER2 expression is heterogeneous in the tumors and varies during the progress of the disease. ${ }^{[9]}$ Almost $20 \%$ of the outcomes were inaccurate. ${ }^{[10]}$

Noninvasive molecular imaging techniques such as Single Photon Emission Computed Tomography (SPECT) and Positron Emission Tomography (PET) provide a reliable method for repetitive investigating the distribution of the receptor in the whole body. ${ }^{[11,12]}$ Compared with the former, PET has higher image resolution and quality. PET scanner can sensitively detect gamma radiation from positron decay of nuclides (e.g., ${ }^{11} \mathrm{C},{ }^{18} \mathrm{~F}$ ). Due to high sensitivity, PET imaging with a trace amount of radiotracers $\left(10^{-6}-10^{-8}\right.$ grams) can accurately measure molecular targets in the living body without perturbing the biological system. PET with specific probes is a benefit for disease diagnosis and monitoring therapeutic response. ${ }^{[13-15]}$ Radiolabeled antibodies have shown promise in identifying the presence of HER2 in the tumor. ${ }^{[16-18]}$ For example, ${ }^{89}$ Zr-trastuzumab PET/CT detected unsuspected HER2-positive metastases in patients with HER2-negative primary breast cancer. ${ }^{[19]}$ It also found lesions in patients with metastatic HER2-positive 
esophagogastric cancer ${ }^{[20]}$. However, the optimal images with favorable contrasts should be acquired several days (35days) after the administration of the antibodies. ${ }^{[19]}$

Affibody, an engineered small protein that originated from the IgG-binding staphylococcal protein A, is an alternative ligand towards HER2. It is an ideal compound for recognizing the desired targets due to ease of chemical synthesis, quick tumor accumulation and rapid blood clearance, etc. ${ }^{21,22]} \mathrm{ZHER}_{2: 342}$ affibody binds explicitly to HER2, and its derivatives have been labeled with PET nuclides $\left({ }^{18} \mathrm{~F},{ }^{68} \mathrm{Ga}\right.$, etc. $) .{ }^{[23-25] 18} \mathrm{~F}$ labeled $\mathrm{ZHER} 2: 342$ analog, ${ }^{18} \mathrm{~F}-\mathrm{FBEM}-$ ZHER $_{2: 342}$ showed specific binding towards HER2 positive tumors and could be a benefit for detecting status of the receptor in response to therapeutic interventions. ${ }^{23,26] 68} \mathrm{Ga}$ labeled HER2 affibody, ${ }^{68} \mathrm{Ga}-\mathrm{ABY}-025$, discriminated HER2 positive and negative metastatic breast tumor in sixteen patients. After the PET/CT scan with the tracer, treatment plans were changed in three patients ${ }^{[27,28]}$.

Besides the above short half-life radionuclide, few HER2 affibodies labeled with other PET isotopes are reported. ${ }^{89} \mathrm{Zr}$ is an attractive commercially available PET radionuclide with a long half-life ( $T_{1 / 2}=78.4$ hours), which allows for imaging of biological processes at late time points. ${ }^{[29]}$ Meanwhile, attachment of ${ }^{89} \mathrm{Zr}$ to desferrioxamine (DFO) chelator coupled in bioactive substance (such as protein, peptides, etc.) can be achieved under mild conditions with excellent stability. ${ }^{\left[{ }^{30}\right]}$ Besides, ${ }^{89} \mathrm{Zr}$-labeled compounds are also ideal surrogates for the corresponding therapeutic ${ }^{90} \mathrm{Y}$-or ${ }^{177} \mathrm{Lu}$ labeled radiopharmaceuticals to calculate dosimetry and plan therapy program in preclinical or clinical studies. ${ }^{\text {[31] }}$

Previous studies found that ${ }^{18} \mathrm{~F}$ or ${ }^{68} \mathrm{Ga}$ labeled modified HER2 affibody with a novel hydrophilic linker, ${ }^{18} \mathrm{FAl} /{ }^{68} \mathrm{Ga}-$ NOTA-Cys-GGGRDN-ZHER 2:342 (denoted as ${ }^{18} \mathrm{FAl} /{ }^{68} \mathrm{Ga}-\mathrm{NOTA}-\mathrm{Cys}-\mathrm{MZHER} 2$ ), owned satisfactory specific tumor uptakes and favorable tumor to muscle and tumor to blood ratios during 4 hours after injection. ${ }^{[32,33]}$ Due to short half-life, further evaluating the properties of the affibody was difficult beyond 12 hours. To better assess the characters of the modified HER2 affibody, the molecule was coupled with a maleimide derivative of desferrioxamine, MAL-DFO, then the resulting compound was radiolabeled with ${ }^{89} \mathrm{Zr}$. The efficiency of the resulting probe, ${ }^{89} \mathrm{Zr}$-DFO-MAL-Cys-MZHER2 targeting HER2, was also investigated in tumor models.

\section{Methods}

Cys-ZHER $_{2: 342}$ and Cys-MZHER2 were purchased from Apeptide Co., Ltd. (Shanghai, China). MAL-DFO was purchased from Macrocyclics (Dallas, TX). [ ${ }^{89} \mathrm{Zr}$ ]-oxalate solution was supplied by Cyclotron VU(Netherlands). Human ovarian cancer cell lines SKOV-3 and breast cancer cell lines MCF-7 were obtained from Cell Bank of Shanghai Institutes for Biological Sciences. Female Balb/c nude mice were pruchased from SLAC Laboratory Animal Co., Ltd., China. Analytic and preparative high-performance liquid chromatography (HPLC) were carried out according to the literatures. ${ }^{[32,33]}$ Radio thin-layer chromatography (TLC) was operated on silica gel impregnated glass fiber sheets and analyzed by a BioScan. Sodium citrate solutions ( $0.1 \mathrm{M})$ was used as solvent systems. Mass spectra was acquired from a Waters LCMS system (Waters, Milford, MA).

\section{Affibody Conjugation}

MAL-DFO $(0.35 \mathrm{mg}, 0.50 \mu \mathrm{mol})$ was dissolved in $2 \mathrm{ml}$ ammonium acetate solution $(2 \mathrm{M}, \mathrm{pH}=7)$ and reacted with CysMZHER2 (3mg, $0.40 \mu \mathrm{mol}$ ) at room temperature overnight. (Figure 1) The product was purified with preparative HPLC, followed by lyophilization, as previously described. ${ }^{[32]}$ Mass spectrometry (MS) measured m/z 8077.5 for [MH] ${ }^{+}$ $\left(\mathrm{C}_{351} \mathrm{H}_{560} \mathrm{~N}_{104} \mathrm{O}_{111} \mathrm{~S}_{2}\right.$, calculated molecular weight 8076.8). 
DFO conjugated affibody, DFO-MAL-Cys-MZHER2,(200 $\mu \mathrm{g}, 25 \mathrm{nmol})$ was dissolved in $30 \mu \mathrm{L}$ deionized water and incubated with $185 \mathrm{MBq}\left[{ }^{89} \mathrm{Zr}\right.$-oxalate in $1 \mathrm{ml} 2 \mathrm{M} \mathrm{Na}_{2} \mathrm{CO}_{3}$ solution $(\mathrm{pH}=4)$ for 20 minutes at room temperature. (Figure 2) After diluted with $10 \mathrm{~mL}$ deionized water, the complex was purified by a Varian BOND ELUT C18 column. After washing with $10 \mathrm{~mL}$ deionized water again, the product was eluted with $0.3 \mathrm{~mL} 10 \mathrm{mM} \mathrm{HCl}$ in ethanol. The solution was diluted with $10 \mathrm{~mL}$ saline and passed through a $0.22-\mu \mathrm{m}$ Millipore filter into a sterile vial. Radio HPLC and TLC were used for quality control.

In vitro stability

Aliquots of ${ }^{89} \mathrm{Zr}$-DFO-MAL-Cys-MZHER2 solutions were incubated with $1 \mathrm{ml}$ human serum or PBS for 48 hours at $37^{\circ} \mathrm{C}$. At the preselected time points, the radiopurity was analyzed by TLC.

\section{Cell lines}

Cells were cultured using RPMI-1640 medium supplemented with 10\% (v/v) heat inactivated fetal bovine serum and grown as a monolayer at $37^{\circ} \mathrm{C}$ in a humidified atmosphere containing $5 \% \mathrm{CO}_{2}$.

\section{Cell uptake studies}

Uptake studies of ${ }^{89}$ Zr-DFO-MAL-Cys-MZHER2 in SKOV-3 cells were performed according to the method described. ${ }^{[32]}$ Cells $\left(1 \times 10^{6} /\right.$ well) were incubated at $37^{\circ} \mathrm{C}$ for various times with $37 \mathrm{KBq}$ labeled affibody in a $0.5 \mathrm{~mL}$ serum-free DMEM medium. The nonspecific binding of the tracer was determined by co-incubation with $5 \mu$ M Cys-ZHER $2: 342$. After washed with chilled PBS, the cell pellets in the tube were obtained by centrifugation and measured using a y-counter (PerkinElmer). The cell uptake was expressed as the percentage of the added activity (\%AD/ $10^{6}$ cells) after decay correction.

\section{Animal Model}

All animal experiments were performed according to the national guidelines and approved by the Ethics Committee of Jiangsu Institute of Nuclear Medicine. Tumor models were established by subcutaneously implanted $5 \times 10^{6}$ SKOV-3 or MCF-7 tumor cells suspended in $0.2 \mathrm{~mL}$ PBS into the shoulder region of mice. When tumor sizes reached 100-300 $\mathrm{mm}^{3}$, the mice were used for the following experiments.

\section{MicroPET Imaging}

PET imaging was performed on a microPET scanner (Siemens Inc.). After anesthetized using isoflurane, the mice bearing tumors were placed in the center of the scanner and injected intravenously with $3.7 \mathrm{MBq}{ }^{89} \mathrm{Zr}$-DFO-MAL-CysMZHER2 in the presence or absence of excessive Cys-MZHER $2: 342(10 \mathrm{mg} / \mathrm{kg}$ body weight) via the lateral tail vein. Static PET images of 10 minutes were performed at selected times after tracer injection. Quantitative analysis was operated using the reported methods. ${ }^{[32,33]}$

\section{Biodistribution}

Mice were injected with $0.74 \mathrm{MBq}$ of the tracer via the tail vein and sacrificed at 1, 4, 8, 18, 24, 48, and 72 hours after administration, respectively. For the blocking study, four mice were coinjected with an excess of Cys-ZHER $2: 342$ (10mg/kg body weight) and killed at 1 hour after administration. Tumor and normal tissues of interest were harvested 
and weighed. The radioactivity uptake in tissues was measured in the $\gamma$-counter and expressed as a percentage of the injected radioactivity per gram of tissue $(\% \mathrm{IA} / \mathrm{g})$.

\section{Autoradiography and Histology}

After microPET imaging, the tumors were harvested and sectioned into slices with $5 \mu \mathrm{m}$ thickness at $-80^{\circ} \mathrm{C}$. Ex vivo autoradiography was conducted using the previous method ${ }^{[34]}$. To determine the intratumoral distribution of the tracer, the slices were placed on a phosphorimaging plate for 1 hour. Phosphorimaging plates were read with a plate reader. Quantitative analysis was carried out using OptiQuant software.

After radioactive decay, the slices were used for routine HE staining and HER2 analysis by immunohistochemistry. The procedures were processed following the published literature ${ }^{[33]}$. An epifluorescence microscope (Olympus, X81, Japan) was used to acquire the corresponding images.

\section{Statistical Analysis}

Statistical analyses were performed using GraphPad Prism. Data were analyzed using the unpaired, 2-tailed Student ttest. Differences at the $95 \%$ confidence level

$(p<0.05)$ were considered to be statistically significant.

\section{Results}

\section{Chemistry}

DFO conjugated affibody was readily prepared with a yield of $50 \%$. The chemical purity of the compound was greater than $90 \%$ determined by analytical HPLC.

\section{Radiolabeling Chemistry}

The non-decay corrected yield for ${ }^{89}$ Zr-DFO-MAL-Cys-MZHER2 was $\quad 90.2 \pm 1.2 \%$, respectively. Purification using C18 columns provided a radiochemical purity of more than $95 \%$. HPLC analysis of the tracer showed one single peak with a retention time of 14 minutes $\varangle$ Figure 3区. ${ }^{89} \mathrm{Zr}$-DFO-MAL-Cys-MZHER2 showed a typical elution profile $(\mathrm{Rf}=0.1-0.3)$ on radio TLC. In comparison, free ${ }^{89} \mathrm{Zr}$-oxalate run into the solvent front $(\mathrm{Rf}=0.8-1.0)$

\section{Stability Studies In Vitro}

${ }^{89} \mathrm{Zr}$-DFO-MAL-Cys-MZHER2 was stable for the investigated periods. No free ${ }^{89} \mathrm{Zr}$-oxalate was found after incubation of the tracer in PBS or serum for two days at $37^{\circ} \mathrm{C}$.

\section{Cell Uptake}

Cell uptake studies are shown in Figure 5. The probe quickly accumulated in SKOV-3 and reached plateaus with $10.23 \pm 0.94 \% \mathrm{AD} / 10^{6}$ cells at 30 minutes incubation. By contrast, the uptake levels were significantly decreased in the presence of excess unlabeled Cys-ZHER $2: 342$ at the same time points $\left(2.35 \pm 0.43 \% \mathrm{AD} / 10^{6} \mathrm{cells}\right)$.

\section{Small-Animal PET Imaging}

MicroPET images of mice were listed in Figure 6 and Figure 7. SKOV-3 xenografts were clearly visualized with good contrast even after 48 hours of administration. The SKOV-3 tumor uptakes of the tracer were $11.97 \pm 2.52 \bigotimes 11.43 \pm 2.51 \rrbracket$ 
$10.09 \pm 2.83 \llbracket 8.52 \pm 1.15 \otimes 7.51 \pm 0.39$ and $4.99 \pm 1.68 \% \mathrm{IA} / \mathrm{g}$ at $1,4,8,10,24$ and 48 hours after administration respectively. In contrast, the radio signals in MCF-7 xenografts were weak. The MCF-7 tumor uptakes were 1.98 $\pm 0.28 \otimes 1.79 \pm 0.29 \bigotimes$ $1.39 \pm 0.14$ and $1.21 \pm 0.10 \% \mathrm{IA} / \mathrm{g}$ at $1,4,8$ and 24 hours after administration, respectively. Presaturation of HER2 in tumors by co-injection of nonlabeled Cys-MZHER $2: 342$ caused a significant reduction of radioactivity accumulation in tumors $(2.18 \pm 0.23 \% \mathrm{IA} / \mathrm{g}$ at 60 minutes postinjection).

It also showed that uptake in the liver was deficient and the highest values were nearly $2 \% \mathrm{IA} / \mathrm{g}$ at 1 hour after injection. Accumulated radioactivities were found in kidneys, which suggested that the affibody is mostly excreted through the renal system and urinary tract.

\section{Biodistribution Studies}

The biodistribution data of ${ }^{89} \mathrm{Zr}$-labeled affibody in mice bearing tumors are presented in Tables 1. Similar with PET imaging, radioactivity concentration in SKOV-3 tumors was higher than those in MCF-7 tumors and other healthy organs except for kidneys. Acclamation in SKOV-3 tumors was $11.27 \pm 1.55 \% \mathrm{IA} / \mathrm{g}$ at 1 hour after administration and maintained $5.80 \pm 0.75 \% \mathrm{IA} / \mathrm{g}$ at 48 hours postinjection. ${ }^{89} \mathrm{Zr}$-DFO-MAL-Cys-MZHER2 uptake in the MCF-7 tumors was $1.76 \pm 0.31 \% \mathrm{IA} / \mathrm{g}$ at 1 hour postinjection. Under block with unlabeled HER2 affibody, the SKOV-3 tumor uptake of the tracer significantly reduced to $1.87 \pm 0.15 \% \mathrm{IA} / \mathrm{g}$ at the same time point.

A rapid washout of radioactivity was noted from receptor-negative tissues except kidney. The uptake ratios of tumor-toblood and tumor to muscle values increased from $8.38 \pm 3.73$ and $17.80 \pm 3.08$ at 1 hour postinjection to $198.00 \pm 12.77$ and $393.50 \pm 38.18$ at 72 hours postinjection in mice bearing SKOV-3 tumors, respectively.

\section{Ex vivo Autoradiography and Histology}

Autoradiography studies showed that higher radioactivity accumulated in the periphery of tumors than those at internal tissues. The ratios of radioactive intensity between the two regions were determined to be $5.03 \pm 0.69$.

The pathological analysis confirmed that the peripheral of tumor tissue grew vigorously, and the HER2 receptor was overexpressed. On the contrary, the internal tumor tissue grew slowly even died with low levels of HER2. The results were consistent with the findings by autoradiography.

\section{Discussion}

Desferrioxamine is a hexadentate chelator used for treating iron overload. It is consisted of three hydroxamate groups and forms a thermodynamically stable complex with zirconium. ${ }^{[35]}$ For site-specific labeling, desferrioxaminemaleimide (MAL-DFO) was successfully introduced into MZHER2 by conjugated the thiol group in cysteine residue with the maleimide. ${ }^{89} \mathrm{Zr}$ attached to DFO-MAL-Cys-MZHER2 under mild conditions with nearly quantitative yields. The radiochemical purity was satisfactory determined by both HPLC and TLC. The absence of radiolysis was detected in PBS and serum during in vitro incubation up 48 hours. The stability was derived from a hexadentate complex structure and familiar with the other ${ }^{89} \mathrm{Zr}$-DFO compounds. It means that ${ }^{89} \mathrm{Zr}$ radiolabeled affibody could be prepared at least one day before preclinical or clinical PET studies.

Similar with ${ }^{18} \mathrm{~F}$ or ${ }^{68} \mathrm{Ga}$ labeled affibody, the ${ }^{89} \mathrm{Zr}$ labeled counterpart specifically binds to HER2-positive SKOV-3 tumor cells by in vitro cell uptake experiments. It meant that coupling the affibody with DFO-MAL could not significantly affect the performance of binding to the receptor. 
To better understand the biology characters of the affibody, in vivo experiments including biodistribution studies and microPET imaging were firstly conducted in SKOV-3 and MCF-7 tumor models, which have been often applied for preclinical evaluation the specificity of radiolabeled HER2 affibody. It revealed that ${ }^{89} \mathrm{Zr}$-DFO-MAL-Cys-MZHER2 rapidly concentrated in SKOV-3 tumors after 1 hour postinjection. The uptake values of ${ }^{89}$ Zr-DFO-MAL-Cys-MZHER2 in SKOV-3 tumors at 1 hour postinjection $(\sim 11 \% \mathrm{IA} / \mathrm{g})$ were similar to reported affibody in the HER2 positive xenografts. For example, the accumulation levels of ${ }^{18} \mathrm{~F}-\mathrm{FBEM}-\mathrm{ZHER}_{2: 342},{ }^{18} \mathrm{FAl}-\mathrm{NOTA}-\mathrm{MAL}-\mathrm{MZHER} 2: 342,{ }^{68} \mathrm{Ga}-\mathrm{ABY}-002,{ }^{68} \mathrm{Ga}-\mathrm{NODA}$ GA-

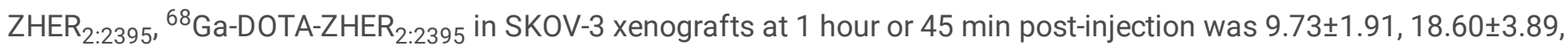
$7.81 \pm 0.70,15 \pm 8$ and $15 \pm 2 \mathrm{ID} \% / \mathrm{g}$ respectively. ${ }^{[26,32,36,37]} \mathrm{Also}$, the corresponding uptake ratios of the tumor to background contrasts such as blood $(8.38 \pm 3.73)$ are also similar with those of ${ }^{18} \mathrm{~F}-\mathrm{FBEM}-\mathrm{ZHER} 2: 342(7.5 \pm 4.5),{ }^{68} \mathrm{Ga}-$ ABY-002(6 \pm 2$),{ }^{68}$ Ga-NODAGA-ZHER $2: 2395(\sim 8)$ and ${ }^{68}$ Ga-DOTA-ZHER $2: 2395(\sim 12)$ respectively. $[26,32,36,37]$ The differences in uptake values or tumor to contrast might be originated from the structure of different chelate agents coupled in the peptides. The uptake in SKOV-3 xenografts was about 10 folds higher than the counter parts of MCF-7 tumors at any time point. Compared with the blood pool, and most of healthy tissues, the retention of the probe remained stable in SKOV-3 tumors over time. Even after 48 hours postinjection, still $40 \%$ of the initially accumulated radioactivity was observed in the SKOV-3 xenografts, and the image contrast was favorable. The uptake ratios of the tumor to blood and tumor to muscle after administration of ${ }^{89} \mathrm{Zr}$-DFO-MAL-Cys-MZHER2 at 72 hours postinjection are nearly 200 and 400, respectively, which are comparable to those of reported ${ }^{18} \mathrm{~F}$ or ${ }^{68} \mathrm{Ga}$ radiolabeled HER2 affibody at

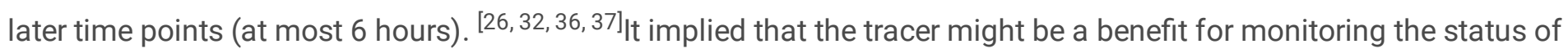
HER2 in tumors with favorable contrast images for long periods at least 72 hours.

The radioactivities distributing in the tumor showed significant heterogeneity by ex vivo immunohistochemistry and autoradiography. Abundant HER2 was expressed in the periphery of cancer and the corresponding radio signal was strong. On the contrary, weak radioactivity was detected in the internal necrotic tissues. Receptor specificity was also confirmed by decreasing the tumor uptake with excessive HER2 affibody. It implied that the targeting property of ${ }^{89} \mathrm{Zr}$ DFO-MAL-Cys-MZHER2 was consistent with the performances of ${ }^{18} \mathrm{~F}$ or ${ }^{68} \mathrm{Ga}$ labeled MZHER2 affibody. ${ }^{[32,33]}$

The uptake values in the liver are comparable to those of ${ }^{18} \mathrm{~F}$, or ${ }^{68} \mathrm{Ga}$ labeled affibody $(\sim 2 \% \mathrm{IA} / \mathrm{g}$ at 1 hour p.i. then decrease to $\sim 1 \% \mathrm{IA} / \mathrm{g}$ at 4 hour p.i.), which suggested that the modification with a hydrophilic linker was effective to decline abdomen background. Similar to other ${ }^{89} \mathrm{Zr}$ labeled affibody such as ${ }^{89} \mathrm{Zr}$-DFO-ZEGFR:2377, higher radioactivity was found in the kidney after blocking. The detailed mechanism was not cleared. The scavenger receptor systems recycling proteins from the urines might mediate the re-absorptions of radiometal labeled peptide in the kidney.

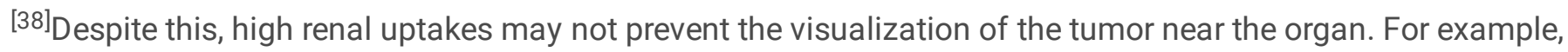
metastases in the adrenal gland were visualized after administration of ${ }^{111}$ In-labeled HER2 affibody, ${ }^{111}$ In-ABY-025 [39]. Low uptakes $(<2 \% \mathrm{IA} / \mathrm{g})$ was observed in the bone. It suggested that the in vivo stability of ${ }^{89} \mathrm{Zr}$-DFO-MAL-Cys-MZHER2 was good since free ${ }^{89} \mathrm{Zr}$ accumulates irreversibly in the mineralized bone.

\section{Conclusion}

A novel PET tracer targeting HER2, ${ }^{89}$ Zr-DFO-MAL-Cys-MZHER2, was successfully prepared by radiolabeling modified affibody with ${ }^{89} \mathrm{Zr}$. The preclinical study revealed that ${ }^{89} \mathrm{Zr}$-DFO-MAL-Cys-MZHER2 is a candidate radiotracer for specific, noninvasive detection of HER2 levels in tumors.

\section{Declarations}


The data generated during the current study are available from the corresponding author on reasonable request.

\section{Acknowledgements}

The authors would like to thank all the participating staff members of Jiangsu Institute of Nuclear Medicine for the study.

\section{Funding}

This work was partially supported by National Natural Science Foundation (81472749,5147307,31671035), National Significant New Drugs Creation Program(2017ZX09304021),Jiangsu Provincial Medical Innovation Team (CXTDA2017024), Jiangsu Provincial Natural Science Foundation (BK20161137هBK20170204囚BE2016632),Jiangsu Health International Exchange Program(JSH-2018-015), Jiangsu talent projects (LGY2017088),Jiangsu Provincial Commission of Health and Family Planning Fundation $₫$ H2017031, QNRC2016628ð,Wuxi Commission of Health and Family Planning Fundation (Q201729).

\section{Author information}

Lizhen Wang contributed equally to this work and share first authorship.

\section{Contributions}

The study design was set up by Yuping Xu, Lizhen Wang, Donghui Pan and Min Yang. Data collection and interpretation was carried out by Lizhen Wang, Xinyu Wang and Yu Liu. Yuping Xu, Junjie Yan and Donghui Pan were responsible for the production of the tracer. The corresponding in vitro and in vivo experiments were performed by Xinyu Wang, Runlin Yang, Mingzhu Li and Yu Liu. Dr Min Yang was the principal investigator of this study. All authors read and approved the final manuscript.

Corresponding author

Correspondence to Min Yang (yangmin@jsinm.org).

\section{Ethics approval}

All animal experiments were performed according to the national guidelines and approved by the Ethics Committee of Jiangsu Institute of Nuclear Medicine.

\section{Consent for publication}

Not applicable

Competing interests

The authors declare that they have no conflict of interest.

\section{Reference}

1. Hanahan D, Weinberg RA. Hallmarks of cancer: the next generation. Cell. 2011;144 (5):646-74.

2. Oh DY, Bang YJ. HER2-targeted therapies-a role beyond breast cancer. Nat Rev Clin Oncol. 2020 ;17(1):33-48. 
3. Pegram MD, Miles D, Tsui CK, Zong Y. HER2-Overexpressing/Amplified Breast Cancer as a Testing Ground for Antibody-Drug Conjugate Drug Development in Solid Tumors. Clin Cancer Res. 2020;26(4)::775-86.

4. Domínguez-Ríos R, Sánchez-Ramírez DR, Ruiz-Saray K, Oceguera-Basurto PE, Almada M, Juárez J, et al. Cisplatinloaded PLGA nanoparticles for HER2 targeted ovarian cancer therapy. Colloid Surf B-Biointerfaces. 2019;178:199207.

5. Mazzotta M, Krasniqi E, Barchiesi G, Pizzuti L, Tomao F, Barba M, et al. Long-term safety and real-world effectiveness of trastuzumab in breast cancer. J Clin Med. 2019;8(2). pii: E254.

6. Bonelli P, Borrelli A, Tuccillo FM, Silvestro L, Palaia R, Buonaguro FM. Precision medicine in gastric cancer. World J Gastroenterol. 2019;11(10):804-29.

7. Eiger D, Pondé NF, de Azambuja E. Pertuzumab in HER2-positive early breast cancer: current use and perspectives. Future Oncol. 2019;15(16):1823-43.

8. Chen Y, Liu L, Ni R, Zhou W. Advances in HER2 testing. Adv Clin Chem. 2019;91:123-62.

9. Jauw YW, O’Donoghue JA, Zijlstra JM, Hoekstra OS, Menke-van der Houven CW, Morschhauser F, et al. 89ZrImmuno-PET: Toward a Noninvasive Clinical Tool to Measure Target Engagement of Therapeutic Antibodies In Vivo. J Nucl Med. 2019;60(12):1825-32.

10. Massicano AV, Lee S, Crenshaw BK, Aweda TA, El Sayed R, Super I, et al. Imaging of HER2 with [89Zr] pertuzumab in Response to T-DM1 Therapy. Cancer Biother Radiopharm. 2019;34(4):209-17.

11. Liang Q, Kong L, Zhu X, Du Y, Tian J. Noninvasive Imaging for Assessment of the Efficacy of Therapeutic Agents for Hepatocellular Carcinoma. Mol Imaging Biol. 2019; doi: 10.1007/s11307-019-01431-5. [Epub ahead of print]

12. Mankoff DA, Farwell MD, Clark AS, Pryma DA. Making molecular imaging a clinical tool for precision oncology: a review. JAMA Oncol. 2017;3(5):695-701.

13. Menon H, Guo C, Verma V, Simone CB. The Role of Positron Emission Tomography Imaging in Radiotherapy Target Delineation. PET Clin. 2020;15 (1):45-53.

14. Provost J, Garofalakis A, Sourdon J, Bouda D, Berthon B, Viel T, et al. Simultaneous positron emission tomography and ultrafast ultrasound for hybrid molecular, anatomical and functional imaging. Nat Biomed Eng. 2018;2 (2):8594.

15. Marcu LG, Moghaddasi L, Bezak E. Imaging of tumor characteristics and molecular pathways with PET: developments over the last decade toward personalized cancer therapy. Int J Radiat Oncol Biol Phys. 2018;102 (4):1165-82.

16. Aluicio-Sarduy E, Ellison PA, Barnhart TE, Cai W, Nickles RJ, Engle JW. PET radiometals for antibody labeling. J Lablled Compd Rad. 2018;61 (9):636-51.

17. Woo S-K, Jang SJ, Seo M-J, Park JH, Kim BS, Kim EJ, et al. Development of 64Cu-NOTA-Trastuzumab for HER2 Targeting: A Radiopharmaceutical with Improved Pharmacokinetics for Human Studies. J Nucl Med. 2019;60 (1):26-33.

18. Moek KL, Giesen D, Kok IC, de Groot DJA, Jalving M, Fehrmann RS, et al. Theranostics using antibodies and antibody-related therapeutics. J Nucl Med. 2017; (Suppl 2):83S-90S.

19. Dehdashti F, Wu N, Bose R, Naughton MJ, Ma CX, Marquez-Nostra BV, et al. Evaluation of [89 Zr] trastuzumabPET/CT in differentiating HER2-positive from HER2-negative breast cancer. Breast Cancer Res Treat. 2018;169 (3):523-30.

20. O'Donoghue JA, Lewis JS, Pandit-Taskar N, Fleming SE, Schöder H, Larson SM, et al. Pharmacokinetics, biodistribution, and radiation dosimetry for 89Zr-trastuzumab in patients with esophagogastric cancer. J Nucl Med. 2018;59 (1):161-6. 
21. De A, Kuppusamy G, Karri VVSR. Affibody molecules for molecular imaging and targeted drug delivery in the management of breast cancer. Int J Biol Macromol. 2018; 107(Pt A): 906-19.

22. Gebauer M, Skerra A. Engineering of binding functions into proteins. Curr Opin Biotechnol. 2019;60:230-41.

23. Kramer-Marek G, Bernardo M, Kiesewetter DO, Bagci U, Kuban M, Omer A, et al. PET of HER2-positive pulmonary metastases with 18F-ZHER2: 342 affibody in a murine model of breast cancer: comparison with 18F-FDG. J Nucl Med. 2012;53 (6):939-46.

24. Yanai A, Harada R, Iwata R, Yoshikawa T, Ishikawa Y, Furumoto S, et al. Site-Specific Labeling of F-18 Proteins Using a Supplemented Cell-Free Protein Synthesis System and 0-2-[18 F] Fluoroethyl-L-Tyrosine:[18 F] FET-HER2 Affibody Molecule. Mol Imaging Biol. 2019;21 (3):529-37.

25. Velikyan I, Schweighöfer P, Feldwisch J, Seemann J, Frejd FY, Lindman H, et al. Diagnostic HER2-binding radiopharmaceutical,[68Ga] Ga-ABY-025, for routine clinical use in breast cancer patients. Am J Nucl Med Mol Imaging. 2019;9 (1):12-23.

26. Kramer-Marek G, Kiesewetter DO, Martiniova L, Jagoda E, Lee SB, Capala J. [18 F] FBEM-Z HER2: 342-Affibody molecule-a new molecular tracer for in vivo monitoring of HER2 expression by positron emission tomography. Eur J Nucl Med Mol Imaging. 2008;35(5):1008-18.

27. Sandström M, Lindskog K, Velikyan I, Wennborg A, Feldwisch J, Sandberg D, et al. Biodistribution and radiation dosimetry of the anti-HER2 affibody molecule 68Ga-ABY-025 in breast cancer patients. J Nucl Med. 2016;57 (6):867-71.

28. Sörensen J, Velikyan I, Sandberg D, Wennborg A, Feldwisch J, Tolmachev V, et al. Measuring HER2-receptor expression in metastatic breast cancer using [68Ga] ABY-025 Affibody PET/CT. Theranostics. 2016;6 (2):262-71.

29. Heskamp S, Raavé R, Boerman O, Rijpkema M, Goncalves V, Denat F. 89Zr-immuno-positron emission tomography in oncology: state-of-the-art 89Zr radiochemistry. Bioconjug Chem. 2017;28(9):2211-23.

30. Brandt M, Cardinale J, Aulsebrook ML, Gasser G, Mindt TL. An overview of PET radiochemistry, part 2: Radiometals. J Nucl Med. 2018;59:1500-6.

31. Dilworth JR, Pascu SI. The chemistry of PET imaging with zirconium-89. Chem Soc Rev. 2018;47 (10):2554-71.

32. Xu Y, Bai Z, Huang Q, Pan Y, Pan D, Wang L, et al. PET of HER2 expression with a novel 18FAl labeled affibody. J Cancer. 2017;8 (7):1170-8.

33. Xu Y, Wang L, Pan D, Yu C, Mi B, Huang Q, et al. PET imaging of a $68 \mathrm{Ga}$ labeled modified HER2 affibody in breast cancers: from xenografts to patients. Br J Radiol. 2019; 92(1104):20190425.

34. Mason C, Kossatz S, Carter L, Pirovano G, Brand C, Guru N, et al. A 89Zr-HDL PET tracer monitors response to a CSF1R inhibitor. J Nucl Med. 2020;61(3):433-6.

35. Raavé R, Sandker G, Adumeau P, Jacobsen CB, Mangin F, Meyer M, et al. Direct comparison of the in vitro and in vivo stability of DFO, DFO* and DFOcyclo* for 89 Zr-immunoPET. Eur J Nucl Med Mol Imaging. 2019;46 (9):196677.

36. Tolmachev V, Velikyan I, Sandström M, Orlova A. A HER2-binding Affibody molecule labelled with 68 Ga for PET imaging: direct in vivo comparison with the 111 In-labelled analogue. Eur J Nucl Med Mol Imaging. 2010;37 (7):1356-67.

37. Altai M, Strand J, Rosik D, Selvaraju RK, Eriksson Karlström A, Orlova A, et al. Influence of nuclides and chelators on imaging using affibody molecules: comparative evaluation of recombinant affibody molecules site-specifically labeled with 68Ga and $111 \mathrm{In}$ via maleimido derivatives of DOTA and NODAGA. Bioconjug Chem. 2013;24(6):11029. 
38. Garousi J, Andersson KG, Mitran B, Pichl M-L, Ståhl S, Orlova A, et al. PET imaging of epidermal growth factor receptor expression in tumours using 89Zr-labelled ZEGFR: 2377 affibody molecules. Int J Oncol. 2016;48 (4):132532.

39. Sörensen J, Sandberg D, Sandström M, Wennborg A, Feldwisch J, Tolmachev V, et al. First-in-human molecular imaging of HER2 expression in breast cancer metastases using the $111 \mathrm{ln}-\mathrm{ABY}-025$ affibody molecule. J Nucl Med. 2014;55(5):730-5.

\section{Table 1}

Table 1 Biodistribution of $\left[{ }^{89} \mathrm{Zr}\right] \mathrm{Zr}-\mathrm{DFO}-\mathrm{MAL}-\mathrm{Cys}-\mathrm{MZHER} 2$ in mice bearing tumors at different time points after administration. ( $\mathrm{n}=4$ per group)

\begin{tabular}{|c|c|c|c|c|c|c|c|c|c|}
\hline \multirow{2}{*}{$\begin{array}{l}\text { Tissues } \\
\text { (\% IA/g) }\end{array}$} & \multicolumn{7}{|c|}{ SKOV-3 } & \multirow{2}{*}{$\begin{array}{c}\begin{array}{c}\text { SKOV-3 } \\
\text { Block }\end{array} \\
\text { 1hours }\end{array}$} & \multirow{2}{*}{$\begin{array}{l}\text { MCF-7 } \\
\text { 1hours }\end{array}$} \\
\hline & 1hours & 4hours & 8hours & 18hours & 24hours & 48hours & 72hours & & \\
\hline blood & $1.47 \pm 0.47$ & $0.52 \pm 0.09$ & $0.24 \pm 0.10$ & $0.16 \pm 0.02$ & $0.09 \pm 0.01$ & $0.04 \pm 0.01$ & $0.02 \pm 0.00$ & $1.03 \pm 0.16$ & $0.88 \pm 0.19$ \\
\hline brain & $0.73 \pm 0.08$ & $0.32 \pm 0.06$ & $0.28 \pm 0.20$ & $0.13 \pm 0.03$ & $0.10 \pm 0.03$ & $0.06 \pm 0.01$ & $0.03 \pm 0.00$ & $0.43 \pm 0.05$ & $0.34 \pm 0.05$ \\
\hline heart & $0.70 \pm 0.24$ & $0.55 \pm 0.04$ & $0.19 \pm 0.08$ & $0.17 \pm 0.05$ & $0.16 \pm 0.07$ & $0.10 \pm 0.03$ & $0.06 \pm 0.01$ & $0.62 \pm 0.14$ & $0.56 \pm 0.06$ \\
\hline liver & $2.03 \pm 0.32$ & $1.39 \pm 0.38$ & $0.63 \pm 0.13$ & $0.30 \pm 0.06$ & $0.17 \pm 0.09$ & $0.09 \pm 0.03$ & $0.05 \pm 0.01$ & $1.84 \pm 0.39$ & $1.65 \pm 0.23$ \\
\hline spleen & $1.47 \pm 0.41$ & $1.00 \pm 0.27$ & $0.68 \pm 0.18$ & $0.28 \pm 0.09$ & $0.20 \pm 0.12$ & $0.10 \pm 0.03$ & $0.06 \pm 0.00$ & $1.12 \pm 0.10$ & $0.92 \pm 0.26$ \\
\hline lung & $1.84 \pm 0.50$ & $1.27 \pm 0.26$ & $0.58 \pm 0.14$ & $0.35 \pm 0.08$ & $0.20 \pm 0.05$ & $0.10 \pm 0.03$ & $0.08 \pm 0.01$ & $0.68 \pm 0.14$ & $1.399 \pm 0.14$ \\
\hline kidney & $101.90 \pm 11.31$ & $145.33 \pm 18.06$ & $141.27 \pm 6.21$ & $146.57 \pm 11.58$ & $135.47 \pm 6.71$ & $128.47 \pm 12.35$ & $131.29 \pm 20.36$ & $175.83 \pm 6.45$ & $149.25 \pm 15.52$ \\
\hline stomach & $1.92 \pm 0.31$ & $1.19 \pm 0.19$ & $0.63 \pm 0.22$ & $0.36 \pm 0.09$ & $0.28 \pm 0.07$ & $0.17 \pm 0.03$ & $0.10 \pm 0.02$ & $1.16 \pm 0.34$ & $1.29 \pm 0.12$ \\
\hline intestine & $1.73 \pm 0.27$ & $1.16 \pm 0.29$ & $0.91 \pm 0.43$ & $0.57 \pm 0.08$ & $0.28 \pm 0.04$ & $0.13 \pm 0.02$ & $0.08 \pm 0.01$ & $1.89 \pm 0.92$ & $0.95 \pm 0.11$ \\
\hline muscle & $0.64 \pm 0.12$ & $0.44 \pm 0.07$ & $0.14 \pm 0.02$ & $0.09 \pm 0.01$ & $0.06 \pm 0.01$ & $0.03 \pm 0.01$ & $0.01 \pm 0.00$ & $0.58 \pm 0.17$ & $0.56 \pm 0.08$ \\
\hline pancreas & $1.49 \pm 0.45$ & $1.52 \pm 0.28$ & $0.82 \pm 0.04$ & $0.56 \pm 0.05$ & $0.38 \pm 0.16$ & $0.15 \pm 0.03$ & $0.06 \pm 0.01$ & $0.62 \pm 0.14$ & $1.38 \pm 0.11$ \\
\hline bone & $1.60 \pm 0.33$ & $1.36 \pm 0.39$ & $0.44 \pm 0.02$ & $0.36 \pm 0.08$ & $0.21 \pm 0.10$ & $0.15 \pm 0.03$ & $0.08 \pm 0.02$ & $0.71 \pm 0.15$ & $0.74 \pm 0.10$ \\
\hline tumor & $11.27 \pm 1.55$ & $10.95 \pm 1.48$ & $11.98 \pm 1.24$ & $9.82 \pm 0.48$ & $8.20 \pm 0.82$ & $5.80 \pm 0.75$ & $3.94 \pm 0.21$ & $2.18 \pm 0.23$ & $1.76 \pm 0.31$ \\
\hline \multicolumn{10}{|l|}{ Ratios } \\
\hline Tumor/blood & $8.38 \pm 3.73$ & $18.28 \pm 2.10$ & $43.09 \pm 3.55$ & $77.06 \pm 14.67$ & $89.00 \pm 12.89$ & $144.13 \pm 17.93$ & $198.00 \pm 12.77$ & $2.15 \pm 0.26$ & $1.98 \pm 035$ \\
\hline tumor/muscle & $17.80 \pm 3.08$ & $21.23 \pm 2.09$ & $79.52 \pm 18.40$ & $127.88 \pm 12.16$ & $138.07 \pm 18.63$ & $257.06 \pm 29.95$ & $393.50 \pm 38.18$ & $3.78 \pm 1.02$ & $3.37 \pm 0.86$ \\
\hline
\end{tabular}

\section{Supplementary Figure Legends}

Figure 1S TLC chromatograms of free $\left[{ }^{89} \mathrm{Zr}\right]-$ oxalate

Figure 2S Maximum intensity projects of mice bearing SKOV-3 (A), MCF-7 xenografts (B) after injection of ${ }^{89} \mathrm{Zr}$-DFOMAL-Cys-MZHER2 without block and mice bearing SKOV-3 xenografts under block (C) respectively. The tumors are indicated by the arrows and circles.

\section{Figures}


ZHER $_{2: 342}$-Asn-Asp-Arg-Gly-Gly-Gly-Cys- $\mathrm{NH}_{2}+$

Cys-MZHER2

DFO-MAL

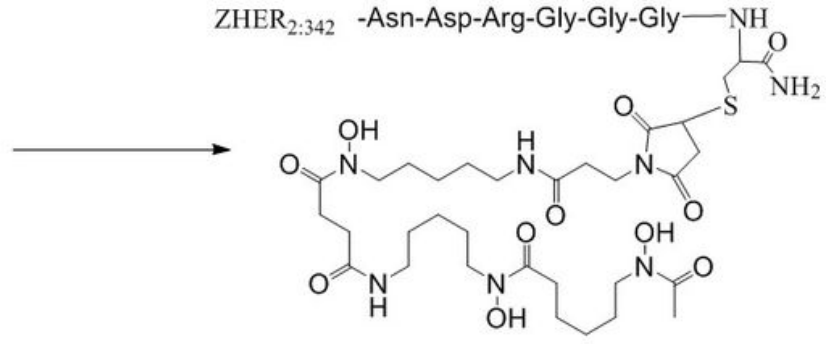

DFO-MAL-Cys-MZHER2

Figure 1

Schemes for synthesis of DFO-MAL-Cys-MZHER2

ZHER $_{2: 342}$-Asn-Asp-Arg-Gly-Gly-Gly-NH

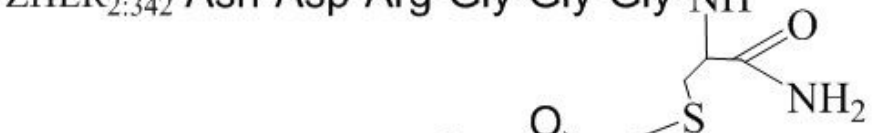

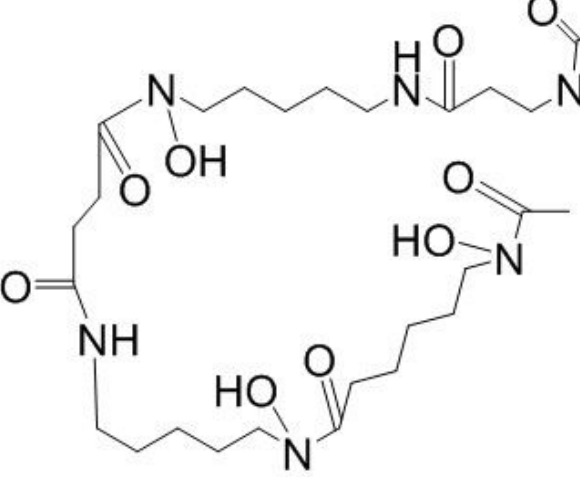

DFO-MAL-Cys-MZHER2
ZHER $_{2: 342}$-Asn-Asp-Arg-Gly-Gly-Gly-NH

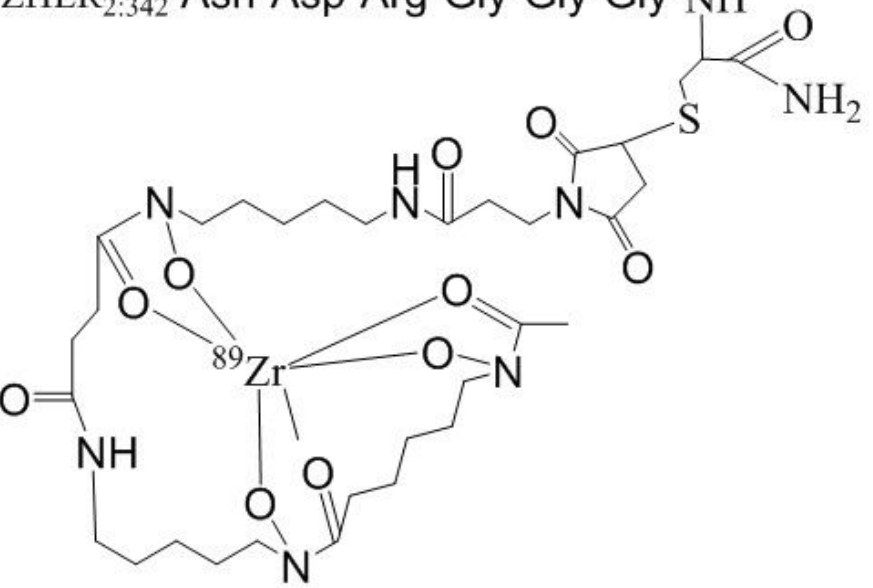

${ }^{89} \mathrm{Zr}-\mathrm{DFO}-\mathrm{MAL}-\mathrm{Cys}-\mathrm{MZHER} 2$

Figure 2

Scheme of radiosynthesis of 89Zr-DFO-MAL-Cys-MZHER2
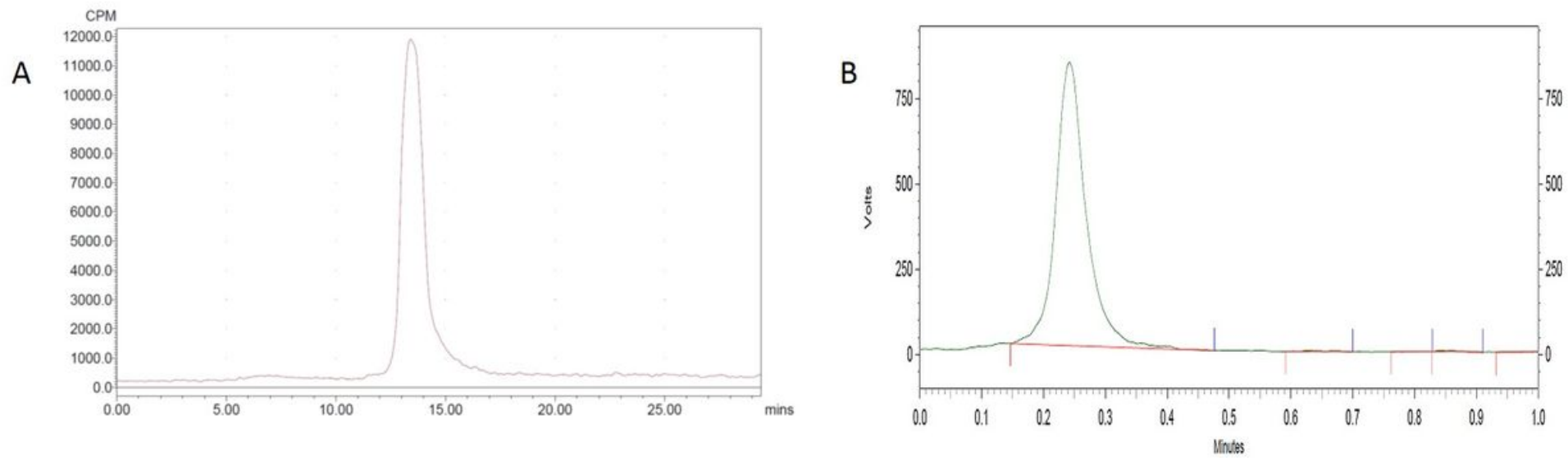

Figure 3

A) HPLC chromatograms of 89Zr-DFO-MAL-Cys-MZHER2; B) TLC chromatograms of 89Zr-DFO-MAL-Cys-MZHER2. 

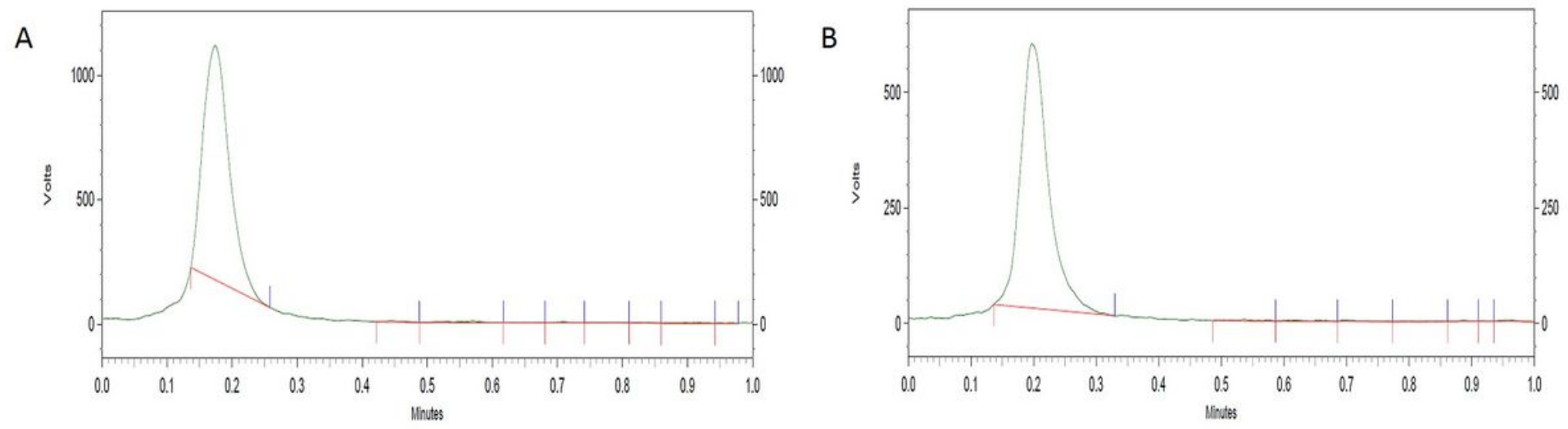

Figure 4

TLC chromatograms of 89Zr-DFO-MAL-Cys-MZHER2 in PBS (A) and serum (B) at $37^{\circ} \mathrm{C}$ for 48 hours respectively.

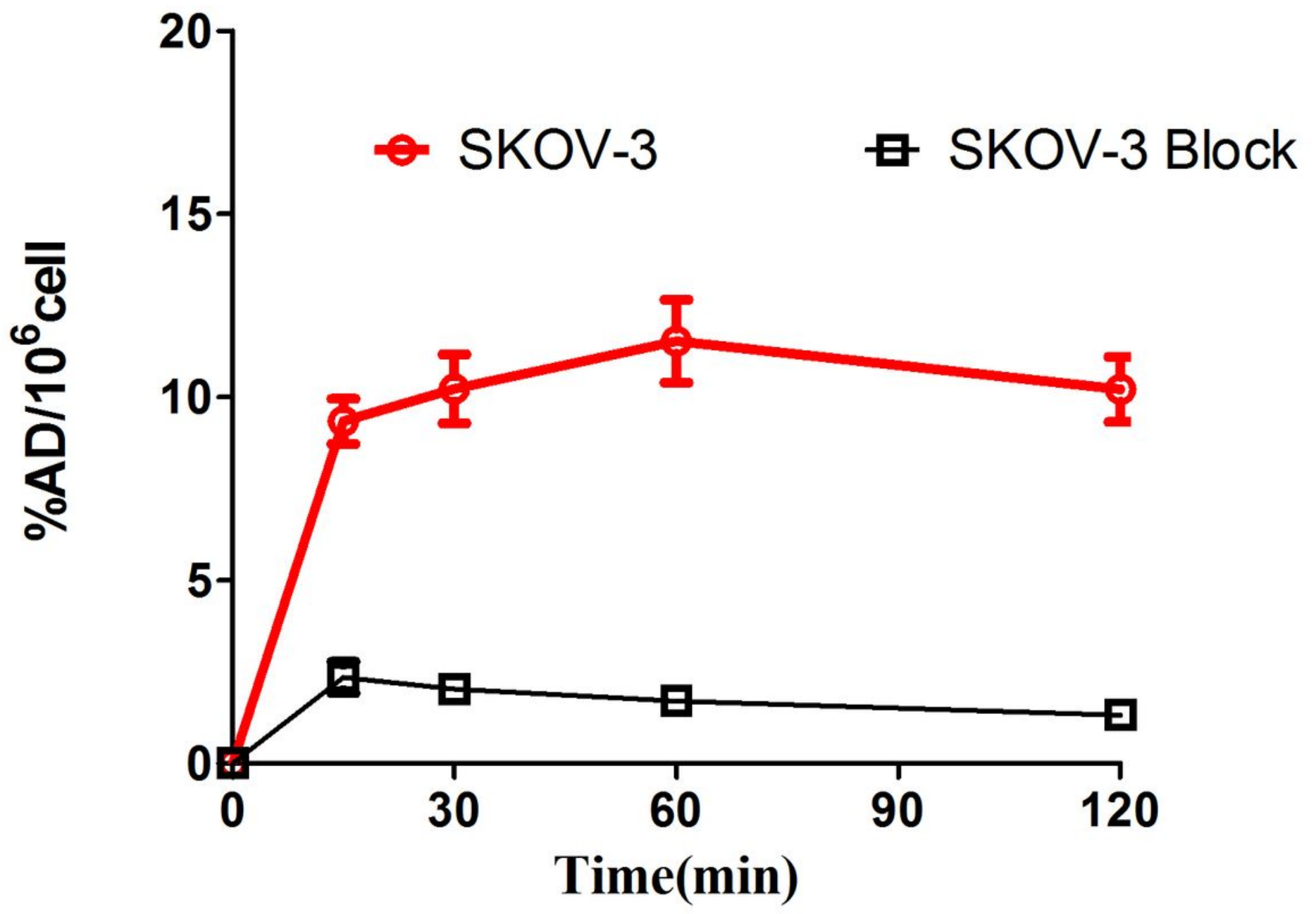

Figure 5

Cell uptake assay of 89Zr-DFO-MAL-Cys-MZHER2 in SKOV-3 cells. 

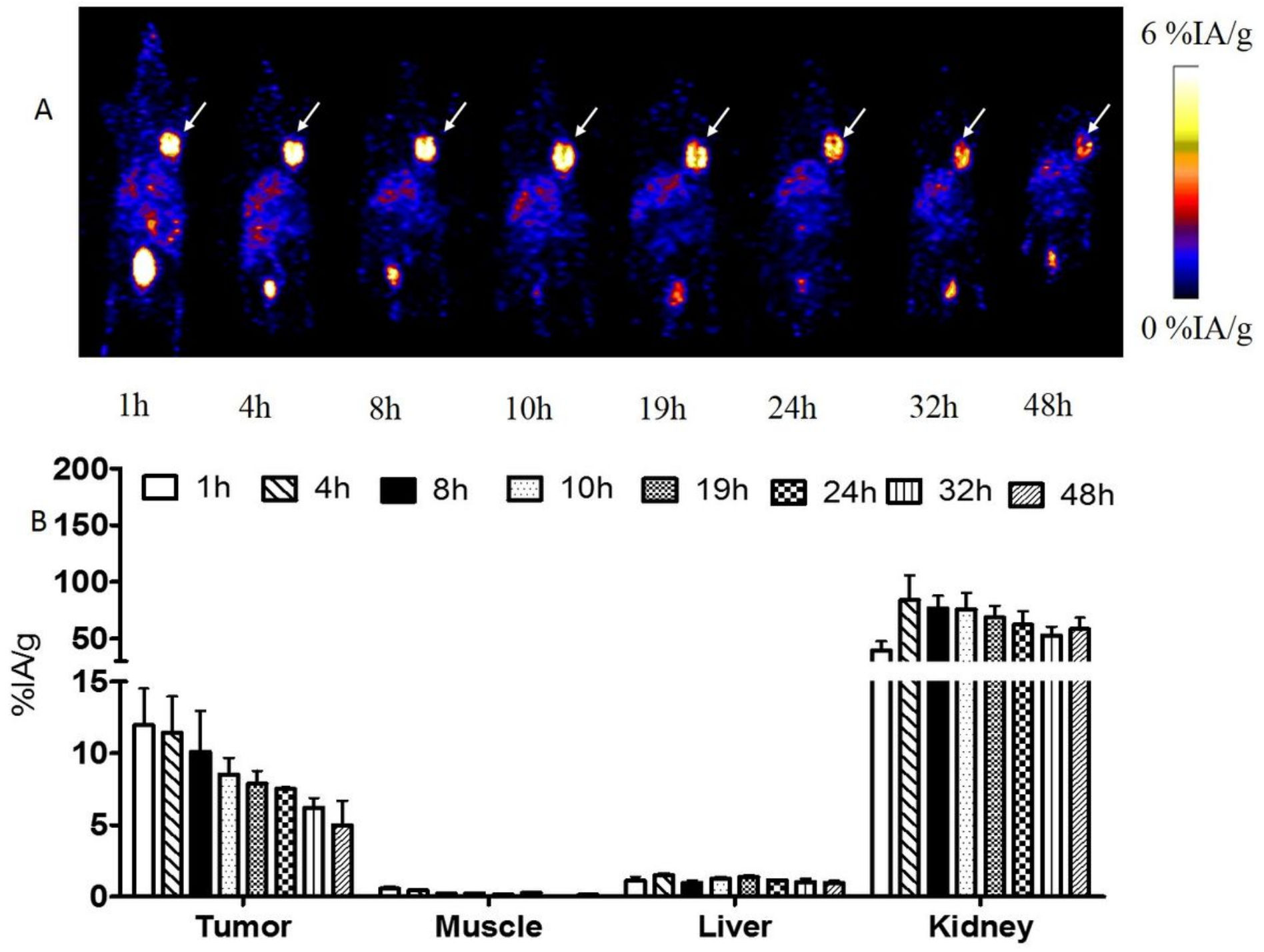

Figure 6

Decay-corrected whole-body PET images of mice bearing SKOV-3 tumors(A) after injection of 89Zr-DFO-MAL-CysMZHER2; B) Quantification of radioactivities in SKOV-3 xenografts models. The tumors are indicated by the arrows. 
A
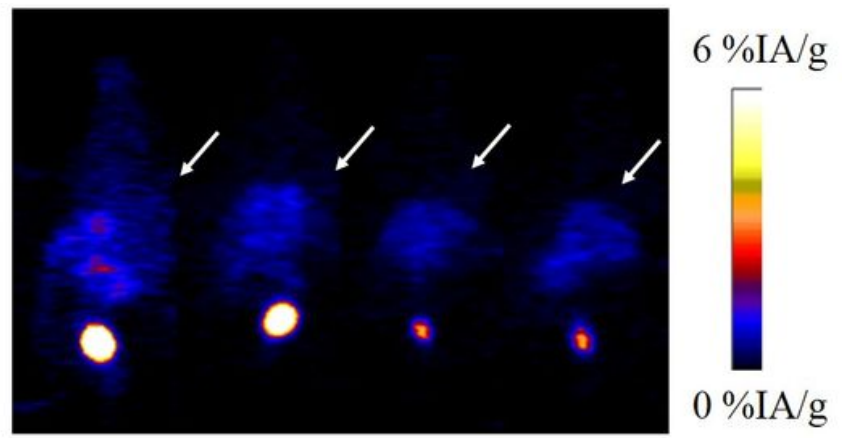

1h $4 \mathrm{~h}$

$8 \mathrm{~h}$

$24 \mathrm{~h}$

B

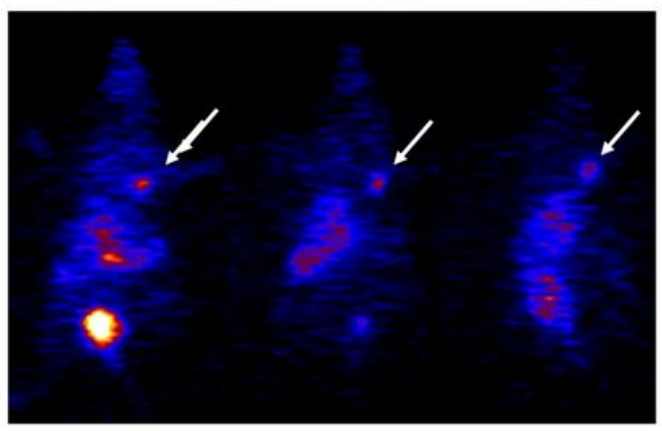

$1 \mathrm{~h}$

$4 \mathrm{~h}$

$8 \mathrm{~h}$

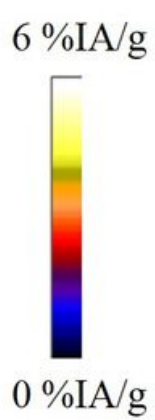

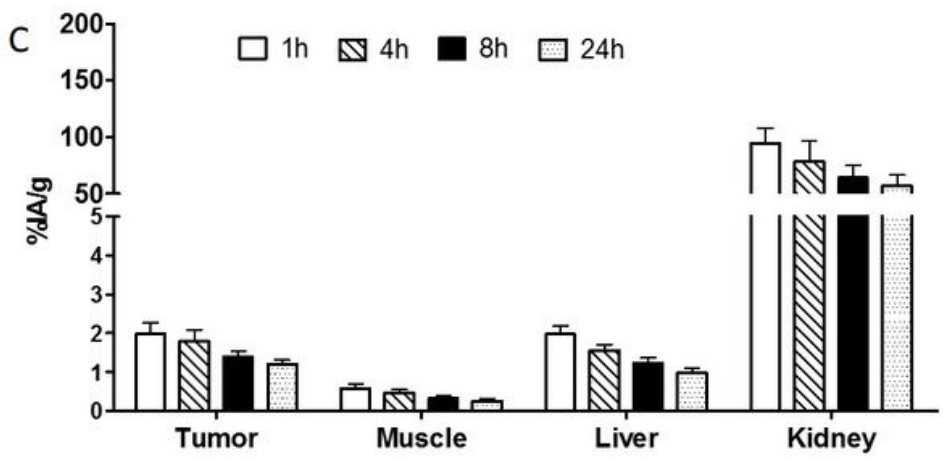

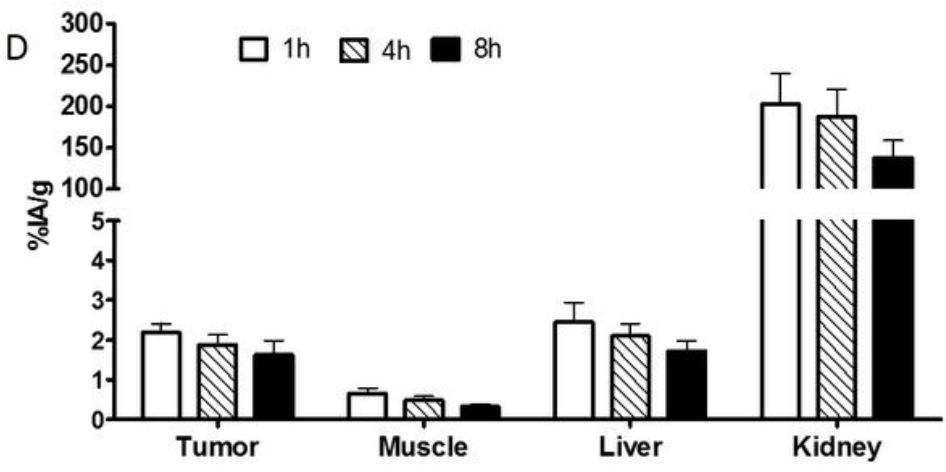

Figure 7

Decay-corrected whole-body PET images of mice bearing MCF-7 xenografts (A) after injection of 89Zr-DFO-MAL-CysMZHER2 ; B) PET images of mice bearing SKOV-3 xenografts after injection of the tracer under block. C) Quantification of 89Zr-DFO-MAL-Cys-MZHER2 in MCF-7 xenografts models and SKOV-3 xenografts models in the presence of excess block agents (D) respectively. The tumors are indicated by the arrows.

A

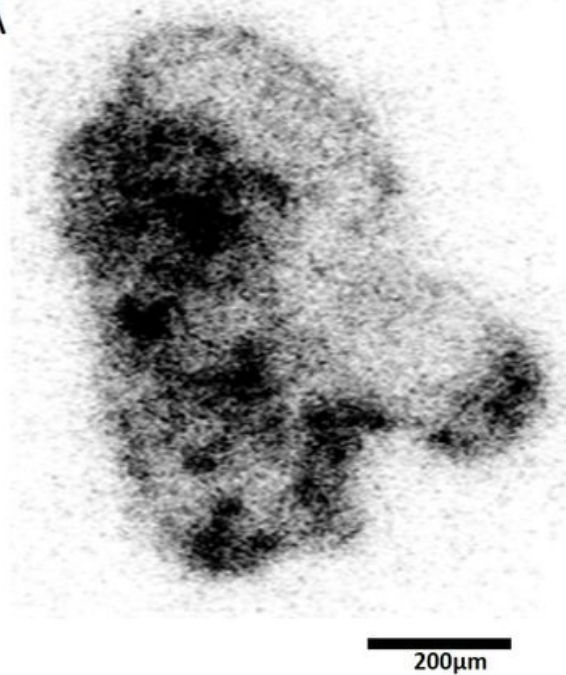

B

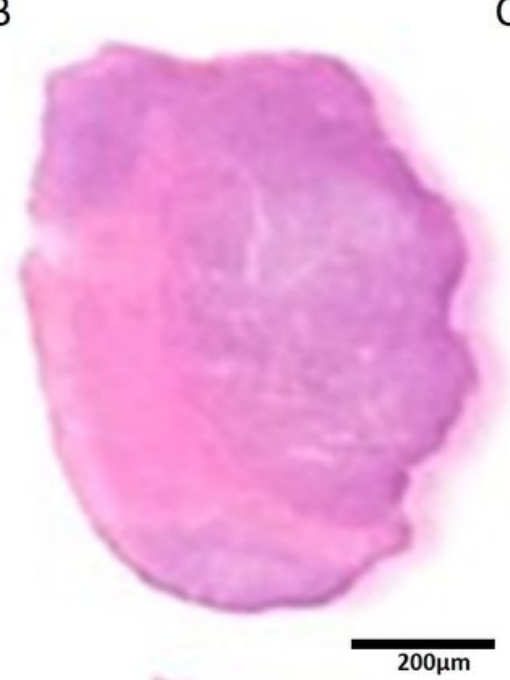

C

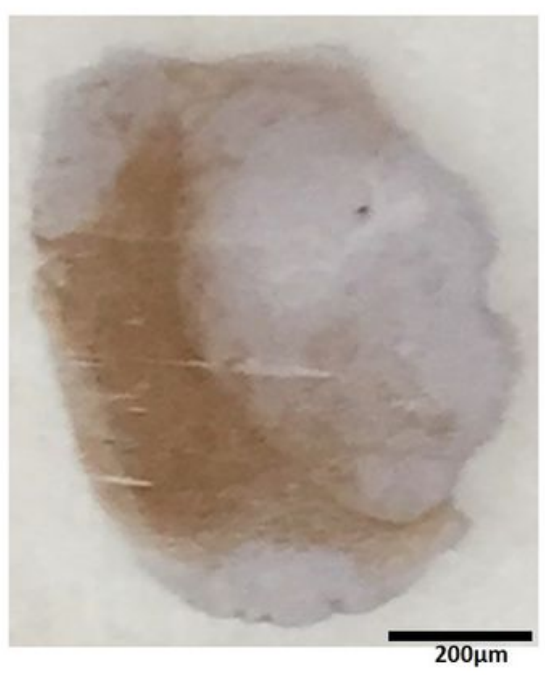

Figure 8 
A) Autoradiographic distribution of 89Zr-DFO-MAL-Cys-MZHER2 in tumor slices. HE staining (B) and immunohistochemical image (C) of HER2 in tumor slices.

\section{Supplementary Files}

This is a list of supplementary files associated with this preprint. Click to download.

- Figure1S.jpg

- Figure2S.jpg 